\title{
Dosis efectiva 90 de oxitocina profiláctica en infusión luego del alumbramiento durante operación cesárea en pacientes en trabajo de parto o cesárea primaria: Un estudio dosis-respuesta con distribución secuencial arriba-abajo.
}

Lavoie A, McCarthy RJ, Wong CA. The ED90 of prophylactic oxytocin infusion after delivery of the placenta during cesarean delivery in laboring compared with nonlaboring women: an up-down sequential allocation dose-response study. Anesthesia and analgesia. 2015 Jul;121(1):159-64.

Análisis crítico: Alberto Pérez E.*, Eduardo Kattan*, Héctor J. Lacassie**. División de Anestesiología, Facultad de Medicina, Pontificia Universidad Católica de Chile. ${ }^{*}$ Residente de Anestesiología. **Profesor Asociado en Anestesiología.

\section{RESUMEN(1)}

Introducción: La administración profiláctica de oxitocina es parte del manejo activo de la tercera etapa del trabajo de parto y reduce el riesgo de hemorragia posparto. Objetivo: estimar con un modelo dosis-respuesta la dosis de oxitocina endovenosa en infusión continua, efectiva en 90\% (ED90) para contracción uterina adecuada luego de trabajo de parto fracasado en pacientes que serán sometidas a operación cesárea versus aquellas sometidas a cesárea electiva. Métodos: Estudio dosis-respuesta, ciego único, de dos ramas utilizando la metodología de la moneda sesgada en relación 9:1 para determinar la DE90. El grupo experimental fueron parturientas sometidas a operación cesárea, secundaria a fracaso en la progresión del trabajo de parto y que habían recibido oxitocina. El grupo control, pacientes sometidas a cesárea electiva. Se les administró oxitocina en infusión continua inmediatamente post nacimiento, de acuerdo a la metodología de la moneda sesgada. Posteriormente, a los 4 minutos del nacimiento, el cirujano estimó si el tono uterino era satisfactorio o no. Los desenlaces secundarios incluyeron requerimientos de uterotónicos adicionales y efectos colaterales maternos. Los datos se analizaron por medio de un modelo de regresión logística y la estimación de la DE90 fue derivada del ajuste de curvas. Resultados: Participaron 38 pacientes sometidas a cesárea electiva y 32 que venían de un trabajo de parto. La DE90 de oxitocina estimada fue significativamente mayor para las pacientes en trabajo de parto $(44,2$ $\mathrm{IU} / \mathrm{h}$, IC95\% 33,8-55,6), comparadas con las sometidas a cesárea electiva (16,2 IU/h (IC95\% 13,1-19,3)). Significativamente más mujeres en el grupo experimental requirieron uterotónicos suplementarios (34\% vs/ $8 \%, p=0,008)$. La incidencia global de efectos colaterales fue mayor en el grupo experimental (69\% vs/ $34 \%, p=0.004)$. Conclusión: La mujeres con exposición previa a oxitocina exógena requieren una mayor dosis de infusión inicial de oxitocina para prevenir atonía uterina post operación cesárea que aquellas sin exposición previa.

\section{ANÁLISIS DE LA INVESTIGACIÓN}

\section{A.Relevancia clínica de la investigación}

La oxitocina es una hormona peptídica neurohipofisiaria, droga de elección para la inducción del trabajo de parto y para lograr la contracción uterina posterior al alumbramiento. Su uso profiláctico ha permitido reducir la incidencia de hemorragia post parto. Cuando se administra rápidamente, la oxitocina provoca hipotensión, 
taquicardia y cambios hemodinámicos significativos que podrían haber contribuido incluso a la muerte de pacientes. Es por ello que se recomienda utilizarla en infusión continua y en dosis ajustadas. Se ha estimado que la dosis efectiva mínima (ED90) de oxitocina en bolo necesaria para producir una adecuada contracción uterina posterior a una cesárea electiva era de 17 UI/hr (IC95\% 9-26)(2). En pacientes embarazadas recibiendo oxitocina endovenosa, que presentan detención en la progresión del trabajo de parto y son sometidas a cesárea, ¿aumentan los requerimientos de oxitocina en infusión continua para lograr la contracción uterina adecuada posterior al alumbramiento?

\section{B.El estudio(1)}

Diseño: Estudio clínico prospectivo, controlado, con un ciego único, realizado entre agosto 2012 a junio 2013 en la Universidad Northwestern en Chicago, EE.UU. Pacientes: 70 embarazadas de término, mayores de 18 años, ASA I-II, no obesas, sometidas a operación cesárea. Intervención: Treinta y dos pacientes con trabajo de parto fracasado que recibieron oxitocina exógena, fueron sometidas a cesárea de urgencia bajo anestesia epidural. Se les administró oxitocina en infusión continua IV post nacimiento, con la metodología de la moneda sesgada (la paciente siguiente recibe una dosis de acuerdo al resultado de la paciente previa en relación 9:1), para detectar la dosis efectiva 90\% (ED90). Comparación: Treinta y ocho pacientes sometidas a cesárea electiva bajo anestesia espinal. Se les administró oxitocina en infusión continua IV post nacimiento, con igual metodología que el grupo experimental. Desenlace primario: Contracción uterina percibida por el cirujano a los 4 minutos del inicio de la dosis de oxitocina. Desenlaces secundarios: Requerimientos de uterotónicos adicionales y efectos colaterales maternos. Resultados: El porcentaje de nulíparas, edad gestacional y con corioamnionitis fue mayor en el grupo de trabajo de parto. Basados en un modelo de regresión logística adaptado a los datos, las DE90 fueron para el grupo experimental de 44,2 IU/h (IC95\% 33,8-55,6) y para el grupo control 16,2 IU/h (IC95\% 13,1-19,3)). Para los desenlaces secundarios, las pacientes en trabajo de parto requirieron más uterotónicos suplementarios $(34 \%$ vs $8 \%, p=0,008)$ y presentan más efectos colaterales ( $69 \%$ vs $34 \%, p=0,004)$.

\section{C.Análisis crítico}

Validez interna: Estudio con descripción apropiada de los criterios de inclusión y exclusión, aleatorio y con seguimiento completo. El tamaño muestral fue calculado y fue apropiado para este tipo de estudios. Los investigadores principales no fueron ciegos a la intervención, pero si lo fue el adjudicador de resultados (cirujano). Es probable que esta falta de ciego sea una fuente de sesgo. La percepción de la adecuación en la contracción uterina es muy subjetivo y depende del cirujano. Finalmente, los grupos no eran equivalentes al inicio, donde las pacientes del grupo experimental fueron operadas bajo anestesia epidural y tuvieron mayor incidencia de corioamnionitis, que es factor de riesgo de atonía uterina. Validez externa: El tipo de pacientes incluidas y la técnica operatoria son similares a la mayoría de las poblaciones mundiales, incluyendo la nuestra. Conclusión: Estudio con riesgo bajo de sesgo, con resultados que pueden ser considerados como una guía biológicamente plausible en que las pacientes en trabajo de parto y que se someten a operación cesárea, tendrán requerimientos aumentados de oxitocina para lograr contracción uterina post operatoria, en un factor de casi 3 veces mayor. La exposición continua de las células miometriales a oxitocina determina una disminución en su capacidad de responder a ella, atribuido a un fenómeno de desensibilización(1).

\section{Palabras clave: Oxitocina; Dosificación; Cesárea; Contracción uterina; Anestesia}

\section{Keywords: Oxytocin; Dosage; Cesarean Section; Uterine Contraction; Epidural anesthesia}

\section{Referencias}

1. Lavoie A, McCarthy RJ, Wong CA. The ED90 of Prophylactic Oxytocin Infusion After Delivery of the Placenta During Cesarean Delivery in Laboring Compared with Nonlaboring Women: An Up-Down Sequential Allocation Dose-Response Study. Anesthesia and analgesia 2015;121:159-64. 2. George RB, McKeen D, Chaplin AC, 
McLeod L. Up-down determination of the $E D(90)$ of oxytocin infusions for the prevention of postpartum uterine atony in parturients undergoing Cesarean delivery. Can J Anaesth 2010;57:578-82. 